\title{
Bandwidth Utilization Prediction in LAN Network Using Time Series Modeling
}

\author{
Ammar T. Namel, Mouayad A. Sahib, Shatha M. Hasan \\ Informatics Institute for Postgraduate Studies, University of Information Technology and Communications, \\ Iraqi Commission for Computers \& Informatics \\ mmmrrr184@gmail.com,mouayad.sahib@uoitc.edu.iq, shathahai@yahoo.co.uk
}

\begin{abstract}
- monitoring the behavior of computer networks is essential for problem identification and optimal management. Part of this behavior to be monitored is the utilization of the network bandwidth. Several techniques are used to model and forecast network traffic such as time series models, modern data mining techniques, soft computing approaches, and neural networks are used for network traffic analysis and prediction. Efficient bandwidth utilization and optimization are very interesting research issues in effective networks because bandwidth is one of the most required and expensive Internet components needed today. It is generally known that the higher the bandwidth available, the better the network performance, thus an essential aid for network design and bandwidth wastage control and a need for traffic models which can capture the characteristics is necessary. In this paper, a time series prediction models were proposed for LAN office network bandwidth utilization. The proposed prediction models are tested by using evaluation metrics used in time series such as MSE and performance evaluation plot. Testing results show that the proposed models can enhance the detection of bandwidth traffic and provide an efficient tool for bandwidth utilization.
\end{abstract}

Index Terms - Dynamic bandwidth allocation, bandwidth consumption, Time Series, ARMA model, AR model.

\section{INTRODUCTION}

Network management is the process of managing resources of a network such as bandwidth, storage, CPU, etc. to enhance the performance of the network. Bandwidth is a very essential, however expensive network resource which must be properly managed to provide the maximum required throughput expected by the network owners and users. Bandwidth can be defined in a variety of ways counting on the context. In computing, bandwidth can be defined as the bit-rate of available or consumed information capacity measured in bits per second (bps). Bandwidth may also be called network bandwidth, data bandwidth or digital bandwidth [1].

Prediction network traffic is of vital profits in various regions, for example, dynamic bandwidth allocation, network security, and network planning and predictive congestion control. In general, two kinds of predictions can be identified: long and short period predictions. Long-term traffic prediction (hours, days, years) offers a detailed estimation of traffic models useful for evaluating future capacity requirements. This evaluation permits for more precise planning and better-controlling decisions. Short period prediction (milliseconds to minutes) is linked to dynamic resource allotment. Short term prediction can be used to enhance Quality of Service (QoS) mechanisms as well as for congestion control and for efficient resource management. It can also be used for routing packets [2]. It is common that bandwidth can be stable or variable. Stable bandwidth equals to an approximate calculation of the 
mean or peak rates. However, variable bandwidth can dynamically be allocated various bandwidth allocation algorithms. Mean rate based bandwidth allocation can cause overcrowding (high loss rate), while the highest rate could lead to a zero loss. It may cause wasting of network resources (low utilization). furthermore, allocating bandwidth based on prediction has shown to be more effective, and these schemes may lead to better management of the network and improved resource allocation [3].

In this paper, a time series models were developed to predict future bandwidth utilization for each user in a certain office network. The datasets are tested for stationarity by ACF plot and ADF test and then the ACF plot and AIC are used for model identification. Performance metrics such as MSE and performance evaluation plot are then applied to the proposed models to examine the quality of fit of the proposed models. Finlay, it is observed that the proposed models can predict short-term future bandwidth consumption for each user and this will help in dynamic bandwidth allocation, congestion avoidance, etc.

The arrangement of the paper is as follows: Section 2 describes related work. The paper methodology is explained in section 3. Discussed results are presented in section 4. Lastly, in section 5, conclusion and suggestions for future work are recommended.

\section{RELATED WORK}

In recent literature, various research methods have been reported on network traffic prediction to avoid performance degradation problems. These methods endeavor to improve the effectiveness and efficiency of the network traffic by monitoring and prediction future data packets flow. One method for achieving this goal is to find a model with accurate traffic prediction. This model will have the capability to catch the important traffic features, such as short and long ranges dependences.

Time series can be divided into linear and nonlinear. Autoregressive (AR) model, Moving Average (MA), Autoregressive Moving Average (ARMA), and Autoregressive Integrated Moving Average (ARIMA) are typical linear time series models. AR, MA, and ARMA are used to model linear and stationary time series, however, ARIMA can be used for modeling non-stationary ones. Nonlinear time series such as the autoregressive conditional heteroscedasticity (ARCH) and generalized autoregressive conditional heteroscedasticity $(\mathrm{GARCH})$ are used to model nonlinear time series.

ARIMA model has been proposed for network traffic prediction in [4]. Authors tested mobile network from Heilongjiang series. Mean Absolute Percentage Error (MAPE) metric have been used to compute and assess forecast outcomes of ARIMA model, using a correlation coefficient function to represent the time series. With this approach, the ARIMA model achieves high prediction. A proposed hybrid model of two-dimensional correction and single exponential smoothing (SES) prediction method presented in [5]. A mobile company was selected for network traffic data collection. The results of this study showed a comparative prediction performance between proposed and classical models. The model proved an improved efficiency in its accuracy of prediction. Neural network Ensemble (NNE) ARIMA and Holt-Winter methods with time series model for prediction Internet traffic were suggested in [6]. The collecting of data based on the TCP/IP protocol for an internet service provider. The models were applied on data at various time intervals ( 5 minutes, 1 hour, and 1 day). To calculate the prediction performance, MAPE metric was used. A comparison between NNE, ARIMA and Holt-Winter methods used for prediction was performed. For one day time intervals, the Holt-Winter method is preferred. However, for shorter time intervals, 5 minutes and 1 hour time periods, the ENN and ARIMA methods provide a better prediction. The NNE was noticed to be more precise in overall. In [7] clustering with Auto-Regressive Moving Average (ARMA) and Weighted Exponential Smoothing (WES) models were integrated into one model to support the prediction of packets loading amount in the network traffic. The dataset of this paper is collected from real network traffic. The experimental outcomes explain that the proposed model can be an efficient method to 
increase forecast efficiency obtained with the aid of clustering. In [8] A prediction approach of network traffic using time series models was proposed. The authors showed that the time series models can predict network traffic and classifying the time series model on a daily basis is a suitable way of prediction. An integrated model that integrates clustering with linear and nonlinear time series predicting models, viz. ARMA, Holt-Trend Exponential Smoothing (HTES), Weighted Exponential Smoothing (WES), Hybrid model (Wavelet with WES), and Auto Regressive Neural Network (NARNET) models to improve predicting of loading packets in the network was proposed in [9]. The practical outcomes illustrate that the integrated model is an efficient technique to improve predicting accuracy achieved with the support of derived centroids. The performance metrics MSE, RMSE, and MAPE are used to assess the results of the above integrated proposed model.

Most of the above-mentioned works does not focus on estimating bandwidth utilization by users in the home or office network. Estimating consumed bandwidth in this network can help in an efficient and effective allocation of bandwidth among users especially in case of limited bandwidth. In this paper, a time series prediction models were proposed for users in an office network to estimate bandwidth utilization. Prediction models can help in advance to forecast bandwidth utilization and this will help in dynamic bandwidth allocation.

\section{METHODOLOGY}

The methodology of applying proposed models is as follow:

\section{A. Network Traffic Collection and Post Processing}

The datasets used for the experiments were collected from daily network activity of Suwera Technical Institute in Wasit governorate in Iraq. This network consists of 10 laptops connected to an access point that provide internet services according to $802.11 \mathrm{Wi}$ Fi standard. Daily network traffic of 30 working days (5 hours each day 9:00 AM -2:00 PM) were captured by using the free and open source packet analyzer (Wireshark). Wireshark enables promiscuous mode in monitoring network in which all the traffic on the network can be observed even the traffic not addressed to that network interface card on the host running Wireshark. In Promiscuous mode, every packet of data sent in the network can be received by a network adapter. In the non-promiscuous mode, when a packet of data is transmitted, all the LAN hosts listen to the data to check if the network address included in the data packet is theirs. Else, the packet is passed onto the next LAN hosts until the host with the valid network address is reached. That host then receives and reads the data. Our experiments require checking only the metadata and not the real data that was downloaded. The detailed number of packets for each IP address (user) that forwarded and received during each connection time is recorded. As mentioned above, the network consists of 10user, therefore the traffic obtained was for 10-user. Depending on the IP address assigned to each user, the traffic can be extracted from originally recorded traffic using software is written in Matlab, finally there exist 10 different sub-dataset one for each user.

The Wireshark application captures the data traffic in milliseconds time intervals for each IP, thus, a massive amount of data was recorded. In order to reduce this amount, the readings of 5 seconds interval are averaged. The final dataset for each user consists of 30 rows (each row represents one day) and 3600 columns represent sample data for that day (monitoring is 5 hours in each day, so there exist 18000 seconds of monitoring, and then average download every 5 seconds is calculated ( $18000 / 5=3600$ sample).

In order to visualize the behavior of each user, in terms of bandwidth consumption, and to get understandable chart the average behavior for each user through 30 -day is plotted. To 
show the bandwidth consumption the average behavior of two random users is shown in figures 1 and 2 .

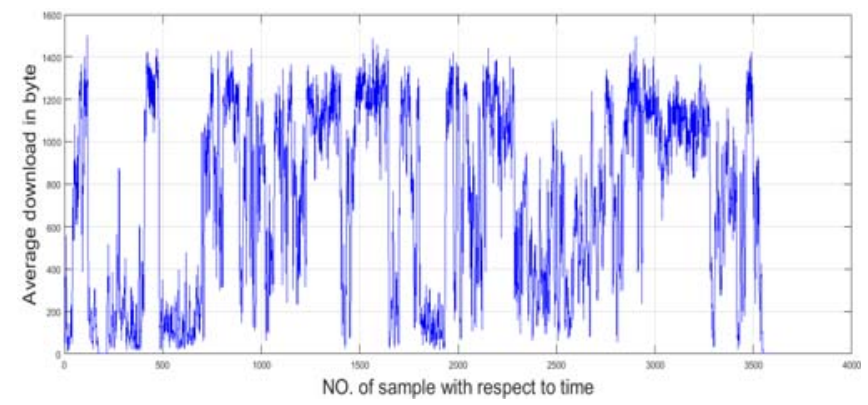

FIG. 1. USER 1 BEHAVIOR IN TERMS OF BANDWIDTH CONSUMPTION

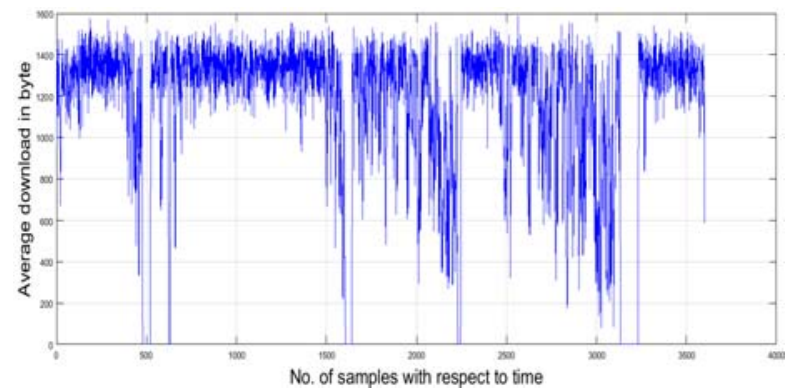

FIG. 2. USER 10 BEHAVIOR IN TERMS OF BANDWIDTH CONSUMPTION

\section{B. Auto Correlation and Partial Auto-Correlation Function}

The Auto-Correlation Function (ACF) calculates the correlation between data point in a series and data points at lags preceding that data point. This information is then aid in computing the (partial autocorrelation function) PACF. ACF and PACF can be used to evaluate the stationary assumption. The ACF is a helpful tool to measure the degree of dependence in identifying the type and the order of the time series model that should be used [10]. The ACF of users 4 and 8 are shown in figures 3 and 4 respectively as an example.
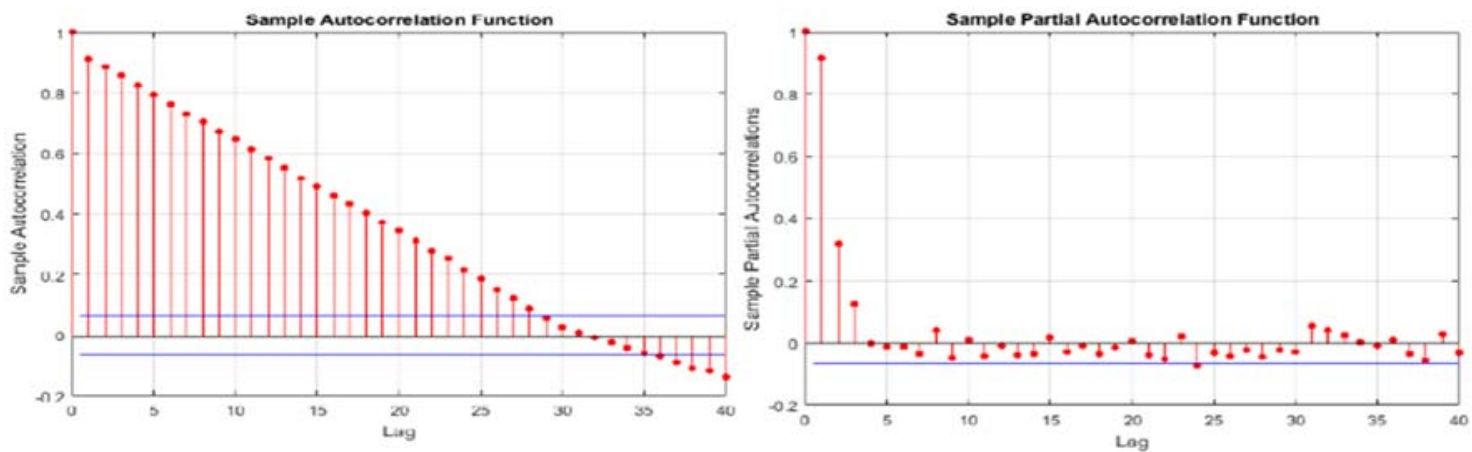

FIG. 3. ACF AND PACF OF USER 4 

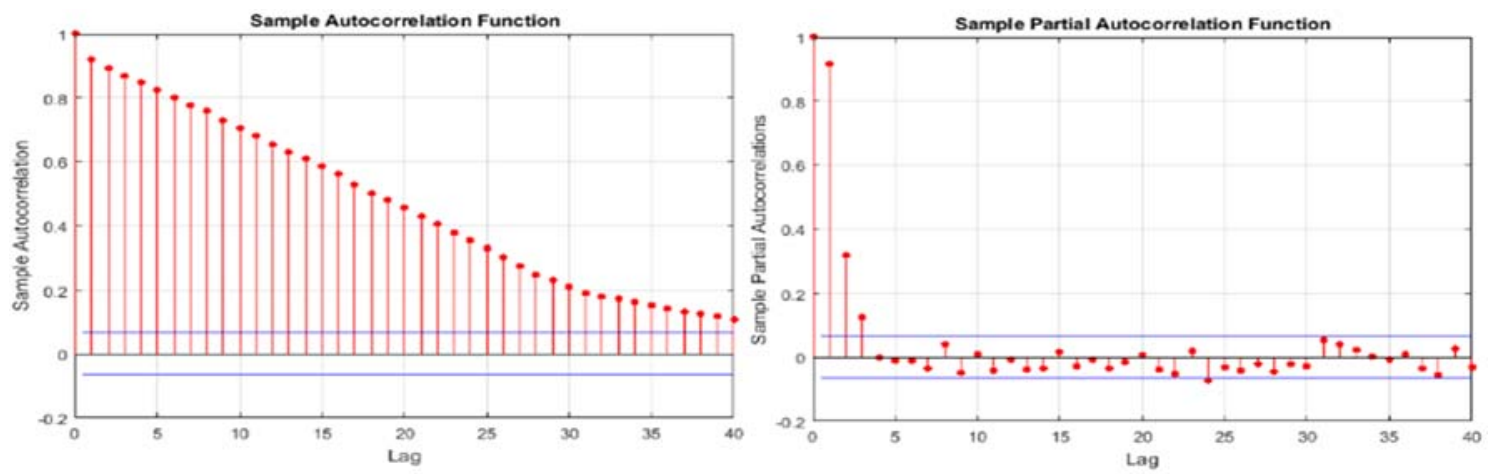

FIG. 4. ACF AND PACF OF USER 8

\section{Stationarity Test of Network Traffic}

A time series data are said to be constant if the basic generation process has a fixed mean and fixed variance with ACF mainly over time. The plotting results of ACF and PACF functions can be interpreted based on general rules. For a stationary time series, the ACF decays to zero relatively speedily, while the ACF of non- constant time series decreases very slowly and the sample PACF cuts off after lag1[11]. A modern statistical test called the Augmented Dickey-Fuller test (ADF) test is also used to confirm the assumptions about the series stationarity [8]. ADF test is one of the statistical unit root tests that is frequently used for determination of series stationarity. Usually, 5\% threshold is being used, what means, that null-hypothesis is unacceptable if the p-value is less than 0.05 [12].

\section{Network Traffic Time Series Modelling}

A time series is a sequence of observations arranged in time [13]. There are many time series model used for modeling Internet traffic these are:

- The Auto-Regressive (AR) model

The autoregressive method comes from the hypothesis that each time observation is linked in a regular and specific way to one or more preceding observations. The mathematical formula for this model is [15]

$$
\mathrm{Z}(\mathrm{t})=\mathrm{c}+\Phi_{1} \mathrm{Z}(\mathrm{t}-1)+\Phi_{2} \mathrm{Z}(\mathrm{t}-2)+\ldots . .+\Phi_{\mathrm{p}} \mathrm{Z}(\mathrm{t}-\mathrm{p})+\varepsilon_{\mathrm{t}}
$$

Where $\mathrm{Z}(\mathrm{t})$ is the time series, $\mathrm{c}$ is a constant, $\varepsilon_{\mathrm{t}}$ is white noise, $\varnothing_{\mathrm{p}}$ is the parameter of $\mathrm{AR}, \mathrm{p}$ is the order of AR model [13].

$$
\text { Here } \varepsilon_{\mathrm{t}}=\frac{1}{\sqrt{2 \pi \sigma^{2}}} e^{\frac{-z_{t}^{2}}{2 \sigma^{2}}}
$$

- The Moving Average (MA) model

The MA is a time series model for modeling univariate time series models. This model has the form:

$$
\mathrm{Z}(\mathrm{t})=\varepsilon_{\mathrm{t}}-\theta_{1} \varepsilon_{\mathrm{t}-1}-\theta_{2} \varepsilon_{\mathrm{t}-2}-\ldots . .-\theta_{\mathrm{q}} \varepsilon_{\mathrm{t}^{-} \mathrm{q}}
$$

where $\mathrm{q}$ is order of moving average, $\theta_{\mathrm{i}}$ is a model parameter, and $\varepsilon \mathrm{t}$ is a white noise[14].

- The Auto-Regressive Moving Average (ARMA) model

An efficient model can be obtained by combining both AR and MA terms. The mathematical formula ARMA model is as follows[15].

$$
\mathrm{Z}(\mathrm{t})=\Phi 1 \mathrm{Z}(\mathrm{t}-1)+\Phi 2 \mathrm{Z}(\mathrm{t}-2)+\ldots . .+\Phi \mathrm{p} \mathrm{Z}(\mathrm{t}-\mathrm{p})+\varepsilon \mathrm{t}-\theta 1 \varepsilon \mathrm{t}-1 \theta 2 \mathrm{\varepsilon t}-2-\ldots . .-\theta \mathrm{q} \varepsilon \mathrm{t}-\mathrm{q}
$$


The terms in the above equation have the same description as given for the AR and MA model.

\section{E. Model Identification}

The initial identifying of a model type can be done with the help of the sample ACF plot to fit an N-length time series. Because sample ACF is symmetrical about lag zero, positive

Lags sample ACF only is plotted from lag one forward to the highest lag of about N/4. The highest order of an AR process can be identified with the aid of the sample PACF plot [16]. Table (1) summarizes ACF and PACF theoretical behavior of ARMA models.

Akaike Information Criterion (AIC) and Bayesian Information Criterion (BIC) are another broadly used measures for identifying the model, where the model with the least AIC or BIC is chosen as the more suitable model. In this paper ACF plot and AIC is used in identifying the more suitable model. The AIC is calculated as $2 \mathrm{~K}-2 \ln$ (L) where $\mathrm{k}$ is the number of free parameters $\mathrm{L}$ is the likelihood function based on the residuals from the model [15].

TABLE 1. ACF AND PACF THEORETICAL BEHAVIOR

\begin{tabular}{ccc}
\hline ACF & PACF & MODEL \\
\hline $\begin{array}{c}\text { Decays exponentially may contain } \\
\text { damped oscillation } \\
\text { q spikes }\end{array}$ & P spikes \\
Both decay exponentially may contain damped oscillation & AR(P) \\
oscillation & MA(q) \\
\end{tabular}

The notation used to describe an AR, MA, ARMA models are the forms AR(p), $\operatorname{MA}(q), \operatorname{ARMA}(p, q)$ respectively, where $p$ is the order of an autoregressive process, $q$ the order of a moving average process [15].

\section{F. Parameter Estimation}

After identifying the proper time series model, the parameter of that model should be estimated. Several methods are used to estimate model parameters such as method of moments, ordinary least square, and Maximum Likelihood Estimation [17]. In this paper Maximum Likelihood Estimation method was used with aid of Matlab mathematical environment to estimate model parameters.

\section{G. Model Validation and Criterion}

After identifying a model and its parameter $\mathrm{s}$ are estimated, the adequacy of the fitted model should be tested, and this can be done with the goodness-of-fit test. In this paper, predictive performance plot checks were used which is a famous strategy used by researchers to perform goodness of fit. Dataset is partitioned into two groups: a training set and a validation set. The training set only is fitted to selected model, and then forecast the fitted model over the validation period. Model forecasts compared against the true, holdout observations, the predictive performance of proposed model can be assessed. Numerical summary of the predictive performance also can be calculated as a prediction mean square error (PMSE). MSE simply refers to the mean of the squared difference between the predicted values and the observed values, MSE is calculated by the following mathematical equation: 


$$
\mathrm{MSE}=\frac{1}{n} \sum_{t=1}^{n} \quad\left(\mathrm{Y}_{\mathrm{t}}-\mathrm{F}_{\mathrm{t}}\right)^{2}
$$

Where $Y_{t}$ is the actual value, $F_{t}$ is the forecasted value, and $n$ is the size of the test set.

When choosing among competing models, the respective PMSE values for competing models are compared. The model with the least PMSE will be selected as the more suitable model [17].

\section{RESULTS AND DISCUSSION}

To fit a suitable model in a time series, it is initially required to examine the dataset for stationarity. Examining sample ACF plot $\mathrm{s}$ confirms stationarity of data, and the result of the ADF test for the raw data of the given datasets confirms the assumption of time series stationarity, where a p-value of all tests are zero rejecting the null hypothesis i.e. the tested series are stationary. For model identification, the sample ACF for all datasets refers initially that the AR model is the more suitable model.

PACF are used in identifying the order $\mathrm{p}$ of an AR model. It is known that several models can be fitted to the same dataset. In this work, four candidate models for each user were employed $(\operatorname{AR}(3), \operatorname{AR}(4), \operatorname{ARMA}(3,2), \operatorname{ARMA}(4,2))$. In order to choose the one that gave the best fit, Akaike's information criterion (AIC) for each fitted model is calculated. The model with the least AIC is the best fit model as shown in table 2 .

TABLE 2. AIC FOR CANDIDATE MODELS

\begin{tabular}{ccccccccccc}
\hline Users & User1 & User2 & User3 & User4 & User5 & User6 & User7 & User8 & User9 & User10 \\
\hline $\operatorname{AR}(3)$ & 15.18 & 13.74 & 9.86 & 8.18 & 2.66 & 6.31 & 2.39 & 9.66 & 18.43 & 34.85 \\
$\operatorname{AR}(4)$ & 15.20 & 13.56 & 9.89 & 8.16 & 2.72 & 6.32 & 2.36 & 9.64 & 18.41 & 34.89 \\
ARMA(3,2) & 15.07 & 14.57 & 9.94 & 8.19 & 2.74 & 6.25 & 2.54 & 10.05 & 18.91 & 34.47 \\
ARMA(4,2) & 14.74 & 14.50 & 9.74 & 8.07 & 2.66 & 6.15 & 2.54 & 9.92 & 18.87 & 34.46 \\
\hline
\end{tabular}

As can be seen from the table (2), AR(4) model is the best fit model for user2, user7, user8, and user9, while ARMA $(4,2)$ is the best fit model for user1, user3, user4, user5, user6, and user10.

For the validation of the efficiency of proposed models for each user, a comparative study with other candidate models should be done such as AR (3), AR (4), ARMA(4,2), and ARMA(4,3). Prediction Mean Square Error (PMSE) for each fitted model is calculated to evaluate the performance of the models. Table (3) shows the calculated PMSE for each fitted model to confirm that the selected model is the more suitable model, (the best model should have least PMSE).

TABLE 3. PMSE FOR CANDIDATE MODELS

\begin{tabular}{ccccccccccc}
\hline Users & User1 & User2 & User3 & User4 & User5 & User6 & User7 & User8 & User9 & User10 \\
\hline $\operatorname{AR}(3)$ & 15.07 & 13.64 & 9.80 & 8.12 & 2.22 & 6.26 & 2.37 & 9.58 & 18.28 & 34.62 \\
$\operatorname{AR}(4)$ & 15.06 & 13.45 & 9.80 & 8.09 & 2.70 & 6.26 & 2.34 & 9.57 & 18.26 & 34.59 \\
ARMA(3,2) & 14.98 & 14.49 & 9.88 & 8.15 & 2.64 & 6.19 & 2.52 & 9.99 & 18.91 & 34.29 \\
ARMA(4,2) & 14.64 & 14.40 & 9.67 & 8.01 & 2.64 & 6.08 & 2.52 & 9.85 & 18.74 & 34.23 \\
\hline
\end{tabular}

The prediction equations and its parameters for each user are expressed in table 4 . 
TABLE 4. PREDICTION EQUATIONS AND ITS PARAMETERS

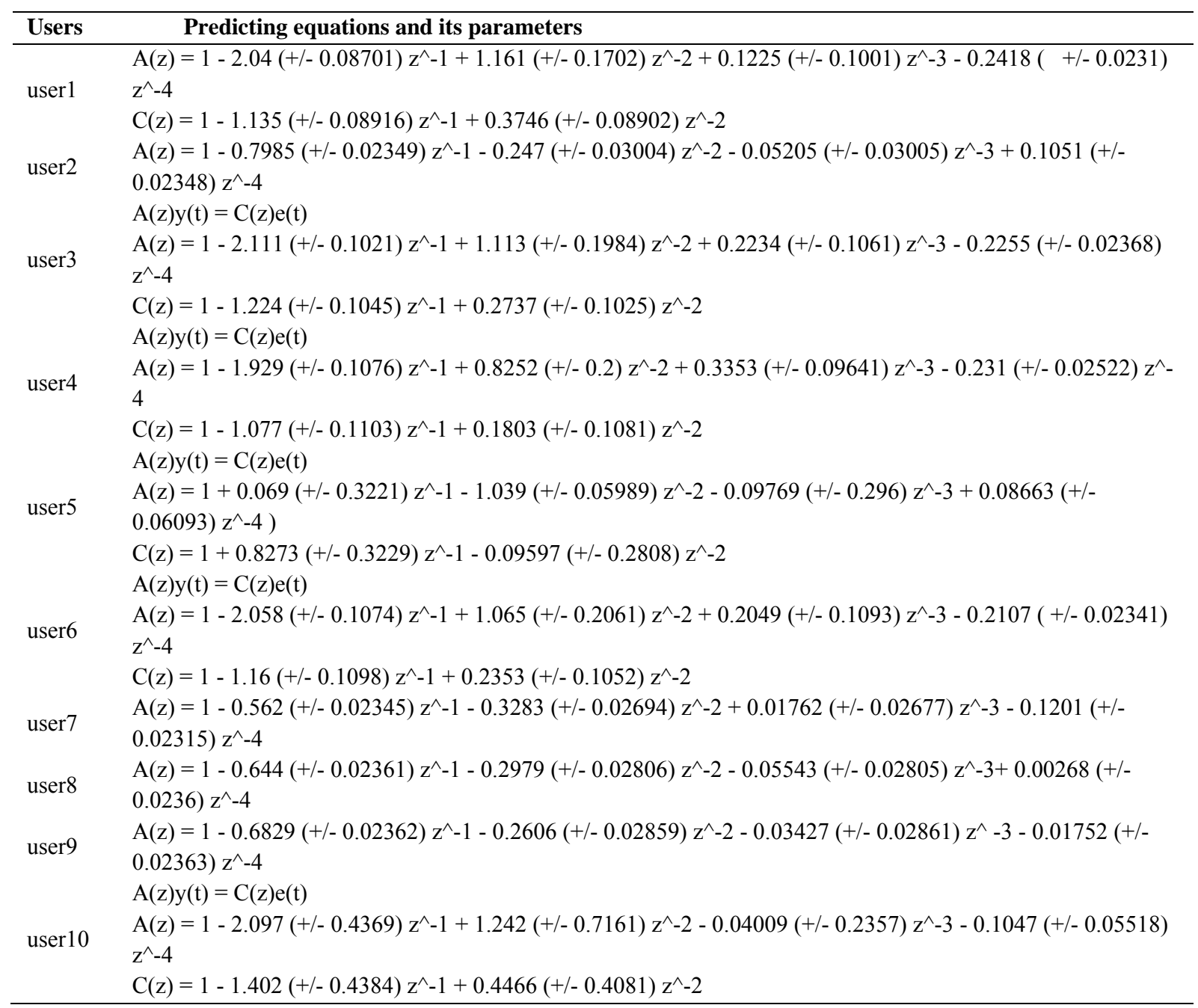

The prediction performance for each fitted model shows that predicted data closely follows original data, the following figures show the plot of prediction performance for fitted models with time interval of 5 seconds. The predicted data (blue), original data (red star).

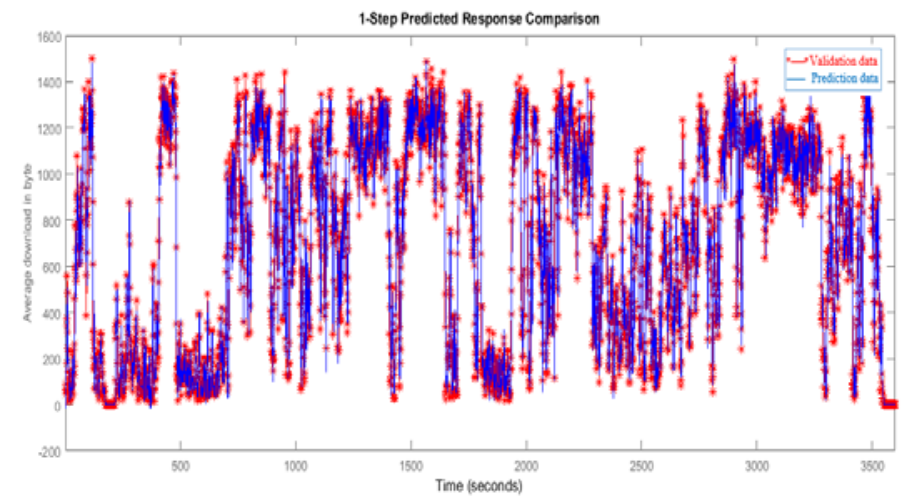

Fig. 5. MODEL (1) PERFORMANCE EVALUATION 


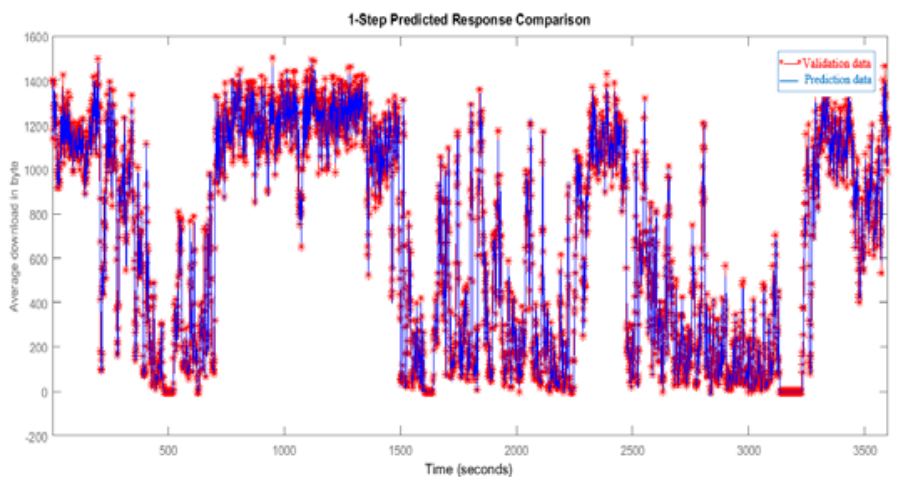

Fig. 6. MODEL (2) PERFORMANCE EVALUATION

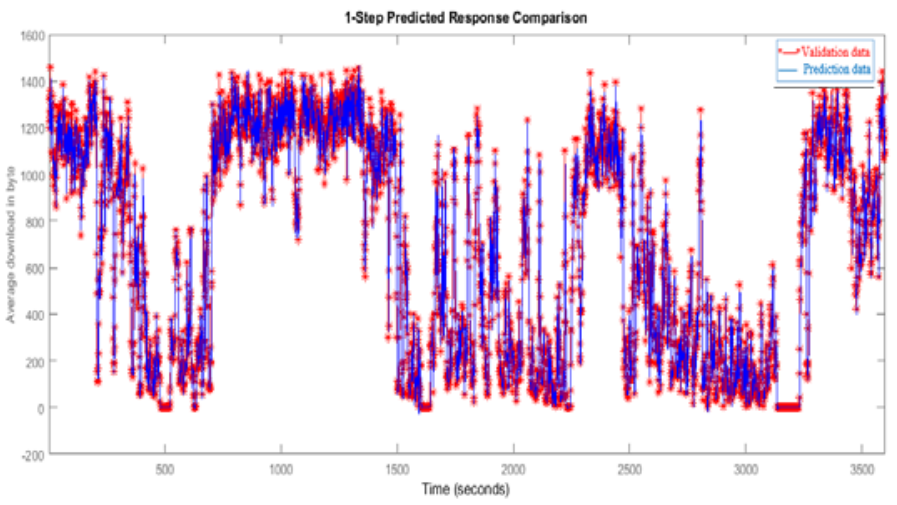

Fig. 7. MODEL (3) PERFORMANCE EVALUATION

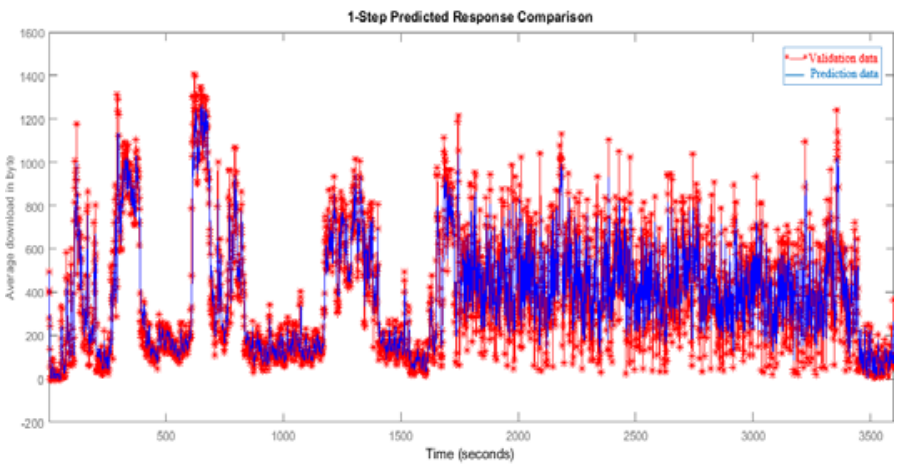

FIG. 8. MODEL (4) PERFORMANCE EVALUATION

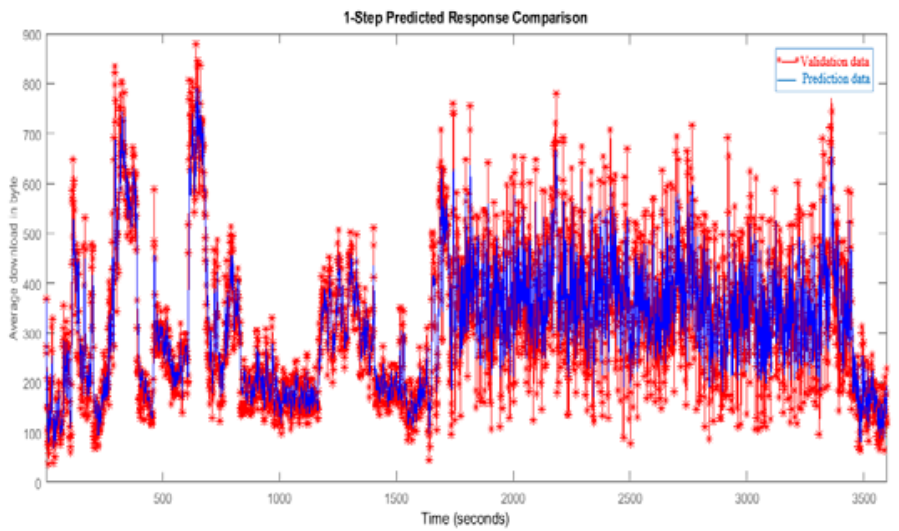

FIG. 9. MODEL (5) PERFORMANCE EVALUATION 


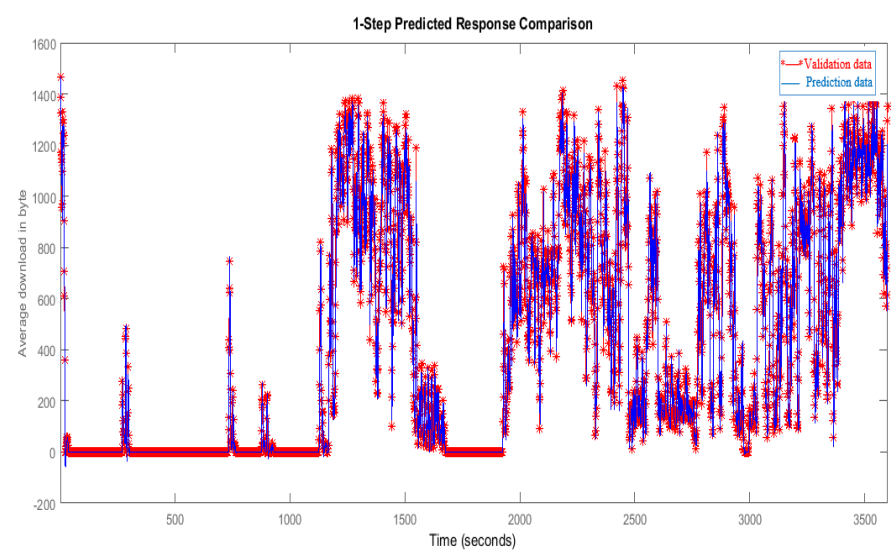

Fig. 10. MODEL (6) PERFORMANCE EVALUATION

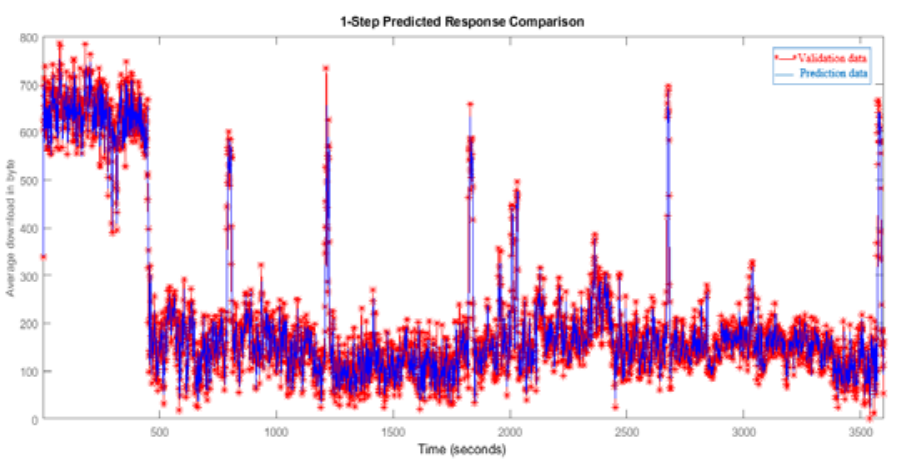

FIG. 11. MODEL (7) PERFORMANCE EVALUATION

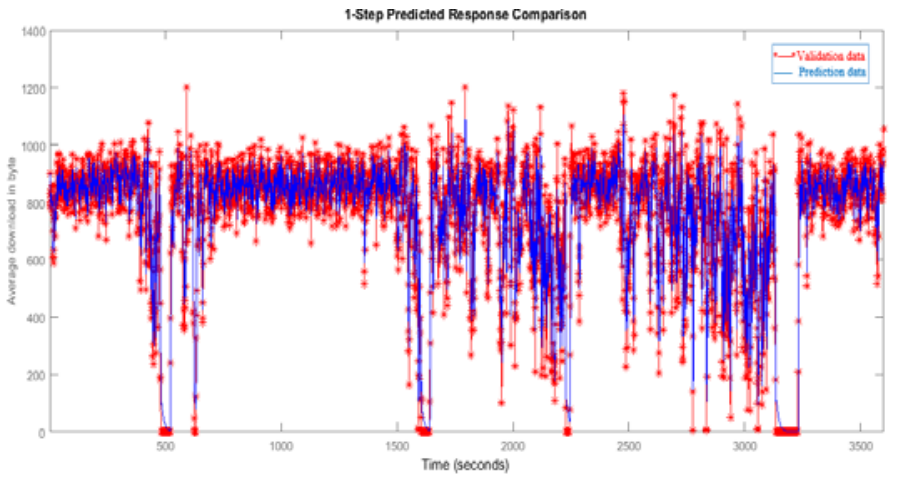

FIG. 12. MODEL (8) PERFORMANCE EVALUATION

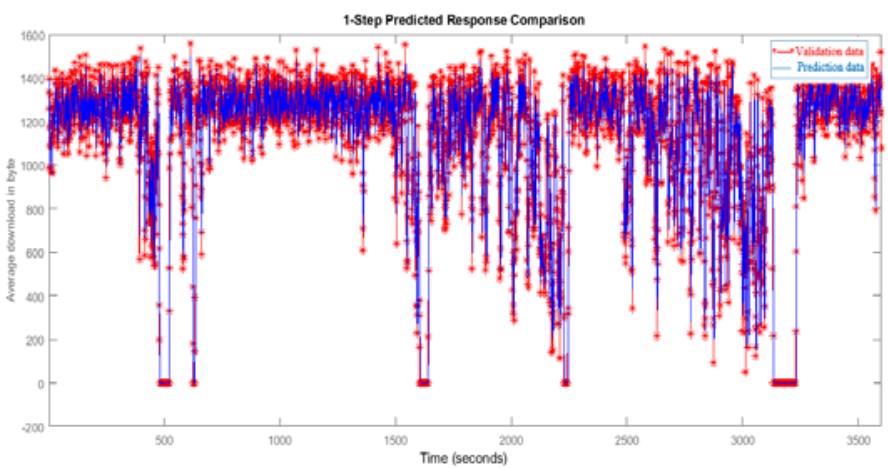

FIG. 13. MODEL (9) PERFORMANCE EVALUATION 


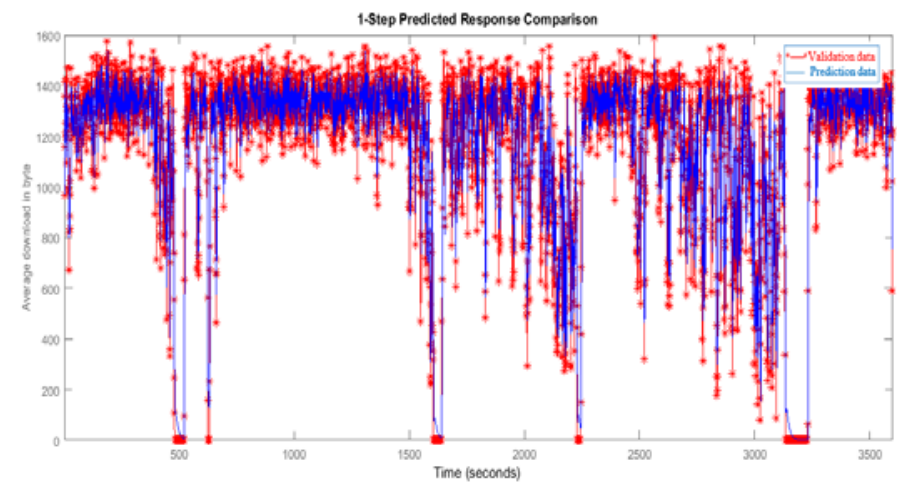

Fig. 14. Model (10) PERFORMANCE EVALUATION

\section{CONCLUSION}

In this paper, a statistical time series models were presented for guessing and predicting network bandwidth consumption on a small office network based on the real-time network monitoring. The traffic was captured using the free and open source application Wireshark. The models can help improve the efficiency of resource utilization such as dynamic bandwidth allocation on high bandwidth networks. Moreover, bandwidth consumption prediction has become substantial for planning and network design. Rapid bandwidth utilization variations make the prediction more challenging. The results of this paper show that the proposed prediction models can efficiently manage the bandwidth utilization of network traffic. A comparison between prediction outcomes was presented between the different candidate models and the proposed models. The PMSE of the proposed models are 15.06, $13.45,9.67,8.01,2.22,6.08,2.34,9.57,18.26$, and 34.23 for model 1 through model 10 respectively. The results display that ARMA models fitted the network traffic data very well and this is assured by the goodness of fit test which is given by the performance evaluation plot. It is noticed that the prediction accuracy of the proposed models is the best compared to the different examined models. In future dynamic bandwidth allocation can be implemented based on the models proposed in this paper to dynamically allocate bandwidth.

\section{REFERENCES}

[1] S. Kim, "Heterogeneous network bandwidth management scheme based on the principal-agent game model," Wireless Networks, vol. 23, pp. 279-287, 2017.

[2] C. Katris and S. Daskalaki, "Dynamic Bandwidth Allocation for Video Traffic Using FARIMA-Based Forecasting Models," Journal of Network and Systems Management, pp. 1-27,2018.

[3] S. Colonnese, F. Cuomo, T. Melodia, and I. Rubin, "A cross-layer bandwidth allocation scheme for HTTP-based video streaming in LTE cellular networks," IEEE Communications Letters, vol. 21, pp. 386-389, 2017.

[4] S. R. Talpur and T. Kechadi, "A Forecasting Model for Data Center Bandwidth Utilization," in Proceedings of SAI Intelligent Systems Conference, 2016, pp. 315-330.

[5] D. W. Yoas and G. Simco, "Using Long-Term Prediction for Web Service Network Traffic Loads," in Information Technology: New Generations (ITNG), 2014 11th International Conference on, 2014, pp. 21-26.

[6] P. Cortez, M. Rio, M. Rocha, and P. Sousa, "Internet traffic forecasting using neural networks," in Neural Networks, 2006. IJCNN'06. International Joint Conference on, 2006, pp. 2635-2642.

[7] T. H. Aldhyani and M. R. Joshi, "An integrated model for prediction of loading packets in network traffic," in Proceedings of the Second International Conference on Information and Communication Technology for Competitive Strategies, 2016, p. 30.

[8] S. Jung, C. Kim, and Y. Chung, "A prediction method of network traffic using time series models," in International Conference on Computational Science and Its Applications, 2006, pp. 234-243.

[9] T. H. Aldhyani and M. R. Joshi, "Integration of time series models with soft clustering to enhance network traffic forecasting," in Research in Computational Intelligence and Communication Networks (ICRCICN), 2016 Second International Conference on, 2016, pp. 212-214. 
[10] Y. Peng, B. Yu, P. Wang, D.-g. Kong, B.-h. Chen, and X.-b. Yang, "Application of seasonal auto-regressive integrated moving average model in forecasting the incidence of hand-foot-mouth disease in Wuhan, China," Journal of Huazhong University of Science and Technology [Medical Sciences], vol. 37, pp. 842-848, 2017.

[11] D. R. Figueiredo, B. Liu, V. Misra, and D. Towsley, "On the autocorrelation structure of TCP traffic," Computer Networks, vol. 40, pp. 339-361, 2002.

[12] S. Basu, A. Mukherjee, and S. Klivansky, "Time series models for internet traffic," in INFOCOM'96. Fifteenth Annual Joint Conference of the IEEE Computer Societies. Networking the Next Generation. Proceedings IEEE, 1996, pp. 611620.

[13] C. You and K. Chandra, "Time series models for internet data traffic," in Local Computer Networks, 1999. LCN'99. Conference on, 1999, pp. 164-171.

[14] Y. Shu, M. Yu, J. Liu, and O. W. Yang, "Wireless traffic modeling and prediction using seasonal ARIMA models," in Communications, 2003. ICC'03. IEEE International Conference on, 2003, pp. 1675-1679.

[15] T. Vafeiadis, A. Papanikolaou, C. Ilioudis, and S. Charchalakis, "Real-time network data analysis using time series models," Simulation Modelling Practice and Theory, vol. 29, pp. 173-180, 2012.

[16] W. Yoo and A. Sim, "Time-series forecast modeling on high-bandwidth network measurements," Journal of Grid Computing, vol. 14, pp. 463-476, 2016.

[17] F. Mehmeti and T. Spyropoulos, "Performance analysis of mobile data offloading in heterogeneous networks," IEEE Transactions on Mobile Computing, vol. 16, pp. 482-497, 2017. 